\title{
MINIMAL APPROXIMATE HESSIANS FOR CONTINUOUSLY GÂTEAUX DIFFERENTIABLE FUNCTIONS
}

\author{
HONGXU LI ${ }^{1}$ and FALUN HUANG ${ }^{1}$
}

(Received 28 June, 2001; revised 1 November, 2001)

\begin{abstract}
In this paper, we investigate minimal (weak) approximate Hessians, and completely answer the open questions raised by V. Jeyakumar and X. Q. Yang. As applications, we first give a generalised Taylor's expansion in terms of a minimal weak approximate Hessian. Then we characterise the convexity of a continuously Gâteaux differentiable function. Finally some necessary and sufficient optimality conditions are presented.
\end{abstract}

\section{Introduction}

Recently, considerable attention has turned to second-order nonsmooth analysis and its applications to optimisation by virtue of various kinds of generalised directional derivatives and generalised Hessians (see for example [1-3, 5-7, 9, 10, 12, 13, 15, 1820]). In particular, much effort has been concentrated on second-order nonsmooth calculus for $C^{1,1}$ functions and optimisation problems involving $C^{1,1}$ functions (see for example $[11,13,17,22,23])$.

In [14] V. Jeyakumar and X. Q. Yang extended optimality condition for $C^{1,1}$ functions to continuously Gâteaux differentiable functions by using approximate generalised Hessians. However, a continuously Gâteaux differentiable function or a $C^{1,1}$ function may admit several approximate generalised Hessians at a point. On the other hand, from the point of view of optimisation, it is important to find approximate generalised Hessians as "small" as possible. So the issue of finding minimal approximate Hessians was raised as an open problem in [14], and the following open questions were given there.

(1) When can the generalised Hessian, $\partial^{\circ \circ} f(x)(u)$ (see the definition in Section 3), be guaranteed as a minimal approximate Hessian for a $C^{1,1}$ function $f$ at $x$ ?

\footnotetext{
'Department of Mathematics, Sichuan University, Chengdu, Sichuan 610064, P. R. China; e-mail: Hoxuli@sohu.com.

(C) Australian Mathematical Society 2004, Serial-fee code 1446-1811/04
} 
(2) Can a minimal approximate Hessian be found for a $C^{1,1}$ function or a continuously Gâteaux differentiable function?

(3) When is the minimal approximate Hessian unique?

The purpose of this work is to investigate minimal approximate Hessians and minimal weak approximate Hessians for continuously Gâteaux differentiable functions, and to answer the above questions.

The outline of this paper is as follows. In Section 2, we give the characterisation of minimal (weak) approximate Hessians for continuously Gâteaux differentiable functions, and a necessary and sufficient condition for the uniqueness of a minimal (weak) approximate Hessian for continuously Gâteaux differentiable functions. Consequently, Questions 2 and 3 above are answered. Then, in Section 3, we prove a necessary and sufficient condition for a generalised Hessian $\partial^{\circ \circ} f(x)(u)$ to be a minimal approximate Hessian for a $C^{1,1}$ function $f$ at $x$. This answers Question 1 above. As applications, in Section 4, we give a modified version of the generalised Taylor's expansion in [14]. Then we characterise the convexity of a continuously Gâteaux differentiable function in terms of a minimal approximate Hessian and a generalised derivative in the sense of Michel-Penot. Moreover, we present some necessary and sufficient optimality conditions for continuously Gâteaux differentiable functions, which modify the results in [14].

\section{Minimal approximate Hessians}

Let $X$ be a Banach space and $X^{*}$ its dual space, and $\langle\cdot, \cdot\rangle$ denote the canonical pair between $X$ and $X^{*}$. Let $f: X \rightarrow R$ be continuously Gâteaux differentiable and $x, u \in X$. The second-order upper Dini-directional derivatives of $f$ at $x$ in the directions $(u, u) \in X \times X$ and $(u,-u) \in X \times X$ (see [14]) are defined, respectively, by

$$
\begin{aligned}
& f_{++}^{D D}(x, u)=\limsup _{s \downarrow 0} \frac{\langle\nabla f(x+s u), u\rangle-\langle\nabla f(x), u\rangle}{s}, \\
& f_{+-}^{D D}(x, u)=\limsup _{s \downarrow 0} \frac{\langle\nabla f(x+s(-u)), u\rangle-\langle\nabla f(x), u\rangle}{s},
\end{aligned}
$$

where $\nabla f(x)$ is the Gateaux derivative of $f$ at $x$. Similarly, second-order lower Dini-directional derivatives of $f$ at $x$ in the directions $(u, u)$ and $(u,-u)$ are defined respectively by

$$
\begin{aligned}
& f_{-+}^{D D}(x, u)=\liminf _{s \downarrow 0} \frac{\langle\nabla f(x+s u), u\rangle-\langle\nabla f(x), u\rangle}{s} \\
& f_{--}^{D D}(x, u)=\liminf _{s \downarrow 0} \frac{\langle\nabla f(x+s(-u)), u\rangle-\langle\nabla f(x), u\rangle}{s} .
\end{aligned}
$$


Note that the first subscript $+(-)$ represents the upper (lower) Dini-directional derivative, and the second subscript $+(-)$ denotes that the second direction is the same as (the negative of) the first direction.

DEFINITION 2.1. A continuously Gâteaux differentiable function $f: X \rightarrow R$ is said to admit an approximate generalised Hessian $\partial^{a a} f(x)(u)$ at $x$ for $u$ if $\partial^{a a} f(x)(u) \neq \emptyset$ is a convex weak* compact set and satisfies

$$
\min \left\{\left\langle x^{*}, u\right\rangle: x^{*} \in \partial^{a a} f(x)(u)\right\} \leq f_{-+}^{D D}(x,-u) \leq \max \left\{\left\langle x^{*}, u\right\rangle: x^{*} \in \partial^{a a} f(x)(u)\right\}
$$

and

$$
\min \left\{\left\langle x^{*}, u\right\rangle: x^{*} \in \partial^{a a} f(x)(u)\right\} \leq f_{++}^{D D}(x, u) \leq \max \left\{\left\langle x^{*}, u\right\rangle: x^{*} \in \partial^{a a} f(x)(u)\right\} .
$$

The function $f$ is said to admit an approximate generalised Hessian at $x$ if for each $u \in X, f$ admits an approximate generalised Hessian at $x$ for $u$. An approximate generalised Hessian $G$ for $f$ at $x$ for $u$ is called a minimal approximate Hessian for $f$ at $x$ for $u$ if for each $\partial^{a a} f(x)(u) \subset G, \partial^{a a} f(x)(u)=G$. Denote such $G$ by $\partial_{m}^{a a} f(x)(u)$.

DEFINITION 2.2. A continuously Gâteaux differentiable function $f: X \rightarrow R$ is said to admit a weak approximate generalised Hessian $\partial^{w \omega} f(x)(u)$ at $x$ for $u$ if $\partial^{w w} f(x)(u) \neq \emptyset$ is a convex weak* compact set and satisfies

$$
f_{++}^{D D}(x,-u), f_{++}^{D D}(x, u) \geq \min \left\{\left\langle x^{*}, u\right): x^{*} \in \partial^{w w} f(x)(u)\right\}
$$

and

$$
f_{-+}^{D D}(x,-u), f_{-+}^{D D}(x, u) \leq \max \left\{\left\langle x^{*}, u\right): x^{*} \in \partial^{w w} f(x)(u)\right\} .
$$

The function $f$ is said to admit a weak approximate generalised Hessian at $x$ if for each $u \in X, f$ admits a weak approximate generalised Hessian at $x$ for $u$. A weak approximate generalised Hessian $G_{w}$ for $f$ at $x$ for $u$ is called a minimal weak approximate Hessian for $f$ at $x$ for $u$ if for each $\partial^{w w} f(x)(u) \subset G_{w}$, we have $\partial^{w w} f(x)(u)=G_{w}$. Denote such $G_{w}$ by $\partial_{m}^{w w} f(x)(u)$.

Since $f_{++}^{D D}(x,-u)=-f_{--}^{D D}(x, u), f_{-+}^{D D}(x,-u)=-f_{+-}^{D D}(x, u)$, the definition of a (weak) approximate generalised Hessian presented here is the same as that in [14]. Clearly, each approximate generalised Hessian of $f$ at $x$ is also a weak approximate generalised Hessian. Furthermore, the following result can be derived immediately from Definitions 2.1 and 2.2.

Proposition 2.1. Let $f: X \rightarrow R$ be continuously Gâteaux differentiable. Then

(i) $f$ admits an approximate generalised Hessian at $x$ for $u$ if and only if both $f_{++}^{D D}(x, u)$ and $f_{-+}^{D D}(x,-u)$ are limited. 
(ii) $f$ admits a weak approximate generalised Hessian at $x$ for $u$ if and only if both $f_{-+}^{D D}(x, u)$ and $f_{-+}^{D D}(x,-u)$ are not $+\infty$, and both $f_{++}^{D D}(x, u)$ and $f_{++}^{D D}(x,-u)$ are not $-\infty$.

(iii) Each single-point (weak) approximate generalised Hessian is a minimal (weak) approximate Hessian.

(iv) $f_{++}^{D D}(x, 0)=f_{-+}^{D D}(x, 0)=0$, and $\left\{x^{*}\right\}$ can be chosen as $\partial_{m}^{a a} f(x)(0)$ and $\partial_{m}^{w \omega} f(x)(0)$ for each $x^{*} \in X^{*}$.

We are now in a position to characterise the minimal (weak) approximate Hessian.

THEOREM 2.1. Suppose that a continuously Gâteaux differentiable function $f$ : $X \rightarrow R$ admits an approximate generalised Hessian at $x$ for $u$.

(i) If $f_{++}^{D D}(x, u)=f_{-+}^{D D}(x,-u)$, then $G(x, u) \subset X^{*}$, is a minimal approximate Hessian for $f$ at $x$ for $u$ if and only if $G(x, u)=\left\{x^{*}\right\}$ for some $x^{*} \in X^{*}$ s.t. $\left\langle x^{*}, u\right\rangle=f_{++}^{D D}(x, u)$.

(ii) If $f_{++}^{D D}(x, u) \neq f_{-+}^{D D}(x,-u)$, then $G(x, u) \subset X^{*}$ is a minimal approximate Hessian for $f$ at $x$ for $u$ if and only if $G(x, u)=\operatorname{co}\left\{x_{1}^{*}, x_{2}^{*}\right\}$ (the convex hull of $\left.\left\{x_{1}^{*}, x_{2}^{*}\right\}\right)$ for some $x_{1}^{*}, x_{2}^{*} \in X^{*}$ s.t. $\left\langle x_{1}^{*}, u\right\rangle=f_{++}^{D D}(x, u)$ and $\left(x_{2}^{*}, u\right\rangle=f_{-+}^{D D}(x,-u)$.

PROOF. (i) If $f_{++}^{D D}(x, u)=f_{-+}^{D D}(x,-u)$, by Definition 2.1, for each $x^{*} \in X^{*}$ s.t. $\left\langle x^{*}, u\right\rangle=f_{++}^{D D}(x, u),\left\{x^{*}\right\}$ is an approximate generalised Hessian for $f$ at $x$ for $u$. Thus, by Proposition 2.1 (iii) $G(x, u)=\left\{x^{*}\right\}$ is a minimal approximate Hessian for $f$ at $x$ for $u$.

On the other hand, if $G(x, u)$ is a minimal approximate Hessian for $f$ at $x$ for $u$, we know from Definition 2.1 that there exists $x^{*} \in G(x, u)$ s.t. $\left\langle x^{*}, u\right\rangle=f_{++}^{D D}(x, u)$, and $\left\{x^{*}\right\}$ is an approximate generalised Hessian for $f$ at $x$ for $u$. So $G(x, u)=\left\{x^{*}\right\}$.

(ii) If $f_{++}^{D D}(x, u) \neq f_{-+}^{D D}(x,-u)$, then for each $x_{1}^{*}, x_{2}^{*} \in X^{*}$ s.t. $\left\langle x_{1}^{*}, u\right\rangle=f_{++}^{D D}(x, u)$ and $\left\langle x_{2}^{*}, u\right\rangle=f_{-+}^{D D}(x,-u)$, it follows from Definition 2.1 that $c o\left\{x_{1}^{*}, x_{2}^{*}\right\}$ is an approximate generalised Hessian for $f$ at $x$ for $u$. Let $\partial^{a a} f(x)(u) \subset c o\left\{x_{1}^{*}, x_{2}^{*}\right\}$ be an approximate generalised Hessian for $f$ at $x$ for $u$. Again by Definition 2.1, there exists $x^{*} \in \partial^{a a} f(x)(u)$ s.t. $\left\langle x^{*}, u\right\rangle=f_{++}^{D D}(x, u)$, then $x_{1}^{*}=x^{*} \in \partial^{a a} f(x)(u)$ since there is only one point in $c o\left\{x_{1}^{*}, x_{2}^{*}\right\}$ satisfying $\left\langle x^{*}, u\right\rangle=f_{++}^{D D}(x, u)$, that is, $x_{1}^{*}$. Similarly we can derive $x_{2}^{*} \in \partial^{a a} f(x)(u)$. Then $\partial^{a a} f(x)(u)=c o\left\{x_{1}^{*}, x_{2}^{*}\right\}$, and hence $G(x, u)=c o\left\{x_{1}^{*}, x_{2}^{*}\right\}$ is a minimal approximate Hessian for $f$ at $x$ for $u$.

On the other hand, if $G(x, u)$ is a minimal approximate Hessian for $f$ at $x$ for $u$, by Definition 2.1 , there exist $x_{1}^{*}, x_{2}^{*} \in G(x, u)$ s.t. $\left\langle x_{1}^{*}, u\right)=f_{++}^{D D}(x, u)$ and $\left\langle x_{2}^{*}, u\right\rangle=f_{-+}^{D D}(x,-u)$. So $\operatorname{co}\left\{x_{1}^{*}, x_{2}^{*}\right\} \subset G(x, u)$. Noticing that $\operatorname{co}\left\{x_{1}^{*}, x_{2}^{*}\right\}$ is an approximate generalised Hessian for $f$ at $x$ for $u$, we have $G(x, u)=c o\left\{x_{1}^{*}, x_{2}^{*}\right\}$. The proof is complete. 
THEOREM 2.2. Suppose that a continuously Gâteaux differentiable function $f$ : $X \rightarrow R$ admits a weak approximate generalised Hessian at $x$ for $u$. Let $A=\left\{x^{*} \in\right.$ $\left.X^{*}: f_{-+}^{D D}(x, u) \leq\left(x^{*}, u\right\rangle \leq f_{++}^{D D}(x, u)\right\}$ and $B=\left\{x^{*} \in X^{*}: f_{-+}^{D D}(x,-u) \leq\right.$ $\left.\left\langle x^{*}, u\right\rangle \leq f_{++}^{D D}(x,-u)\right\}$.

(i) If $A \cap B \neq \emptyset, F(x, u) \subset X^{*}$ is a minimal weak approximate Hessian of $f$ at $x$ for $u$ if and only if $F(x, u)=\left\{x^{*}\right\}$ for some $x^{*} \in A \cap B$.

(ii) If $A \cap B=\emptyset$, we have two cases:

(a) If $f_{++}^{D D}(x, u)<f_{-+}^{D D}(x,-u)$, then $F(x, u)$ is a minimal weak approximate Hessian for $f$ at $x$ for $u$ if and only if $F(x, u)=\operatorname{co}\left\{x_{1}^{*}, x_{2}^{*}\right\}$ for some $x_{1}^{*}, x_{2}^{*} \in X^{*}$ s.t. $\left\langle x_{1}^{*}, u\right\rangle=f_{++}^{D D}(x, u)$ and $\left(x_{2}^{*}, u\right\rangle=f_{-+}^{D D}(x,-u)$.

(b) If $f_{++}^{D D}(x,-u)<f_{-+}^{D D}(x, u)$, then $F(x, u)$ is a minimal weak approximate Hessian for $f$ at $x$ for $u$ if and only if $F(x, u)=\operatorname{co}\left\{x_{1}^{*}, x_{2}^{*}\right\}$ for some $x_{1}^{*}, x_{2}^{*} \in X^{*}$ s.t. $\left\langle x_{1}^{*}, u\right\rangle=f_{++}^{D D}(x,-u)$ and $\left\langle x_{2}^{*}, u\right\rangle=f_{-+}^{D D}(x, u)$.

PROOF. (i) If $A \cap B \neq \emptyset$, it follows from Definition 2.2 that $\left\{x^{*}\right\}$ is a weak approximate generalised Hessian for $f$ at $x$ for $u$ for each $x^{*} \in A \cap B$. Thus $F(x, u)=\left\{x^{*}\right\}$ is a minimal weak approximate Hessian for $f$ at $x$ for $u$ since $\left\{x^{*}\right\}$ is single-point.

On the other hand, if $F(x, u)$ is a minimal weak approximate Hessian for $f$ at $x$ for $u$, it follows from Definition 2.2 that there exists $x^{*} \in F(x, u)$ s.t. $x^{*} \in A \cap B$. So $F(x, u)=\left\{x^{*}\right\}$ since $\left\{x^{*}\right\}$ is a weak approximate generalised Hessian for $f$ at $x$ for $u$.

(ii) (a). If $f_{++}^{D D}(x, u)<f_{-+}^{D D}(x,-u)$, then for each $x_{1}^{*}, x_{2}^{*} \in X^{*}$ s.t. $\left\langle x_{1}^{*}, u\right\rangle=$ $f_{++}^{D D}(x, u)$ and $\left\langle x_{2}^{*}, u\right\rangle=f_{-+}^{D D}(x,-u)$, we know that $c o\left\{x_{1}^{*}, x_{2}^{*}\right\}$ is a weak approximate generalised Hessian for $f$ at $x$ for $u$ by Definition 2.2. Let $\partial^{w w} f(x)(u) \subset \operatorname{co}\left\{x_{1}^{*}, x_{2}^{*}\right\}$ be any weak approximate generalised Hessian for $f$ at $x$ for $u$. Again by Definition 2.2, there exists $x^{*} \in \partial^{w w} f(x)(u)$ s.t. $\left\langle x^{*}, u\right\rangle=f_{++}^{D D}(x, u)$. But there is only one point in $c o\left\{x_{1}^{*}, x_{2}^{*}\right\}$ satisfying $\left\langle x^{*}, u\right\rangle=f_{++}^{D D}(x, u)$, that is, $x_{1}^{*}$, so $x_{1}^{*}=x^{*} \in \partial^{w w} f(x)(u)$. Similarly we can derive $x_{2}^{*} \in \partial^{w w} f(x)(u)$, which implies $\partial^{w w} f(x)(u)=c o\left\{x_{1}^{*}, x_{2}^{*}\right\}$. Hence $F(x, u)=\operatorname{co}\left\{x_{1}^{*}, x_{2}^{*}\right\}$ is a minimal weak approximate Hessian for $f$ at $x$ for $u$.

On the other hand, if $F(x, u)$ is a minimal weak approximate Hessian for $f$ at $x$ for $u$, there exist $x_{1}^{*}, x_{2}^{*} \in G(x, u)$ s.t. $\left\langle x_{1}^{*}, u\right\rangle=f_{++}^{D D}(x, u)$ and $\left\langle x_{2}^{*}, u\right\rangle=f_{-+}^{D D}(x,-u)$, but $\operatorname{co}\left\{x_{1}^{*}, x_{2}^{*}\right\}$ is a weak approximate generalised Hessian for $f$ at $x$ for $u$, so $F(x, u)=$ $\operatorname{co}\left\{x_{1}^{*}, x_{2}^{*}\right\}$.

(b) The proof in this case is similar to (a) and so is omitted. The proof is complete.

REMARK 2.1. (i) By Theorems 2.1 and 2.2, we can see easily that there exists a minimal (weak) approximate Hessian $\left(\partial_{m}^{w w} f(x)(u)\right) \partial_{m}^{a a} f(x)(u)$ in each (weak) approximate generalised Hessian $\left(\partial^{w w} f(x)(u)\right) \partial^{a a} f(x)(u)$ for a continuously Gâteaux differentiable function $f$ at $x$ for $u$. Moreover, each $\left(\partial_{m}^{w w} f(x)(u)\right) \partial_{m}^{a a} f(x)(u)$ is a compact set of a one-dimensional subspace of $X^{*}$. 
(ii) By Proposition 2.1 (i), (ii) and Theorems 2.1 and 2.2, a continuously Gâteaux differentiable function admits a minimal (weak) approximate Hessian if and only if it admits a (weak) approximate generalised Hessian. In particular, a $C^{1,1}$ function, that is, a Gâteaux differentiable function with locally Lipschitz derivative, admits a minimal (weak) approximate Hessian at each $x \in X$ for each $u \in X$, since $f_{++}^{D D}(x, u)$, $f_{++}^{D D}(x,-u), f_{-+}^{D D}(x, u)$ and $f_{-+}^{D D}(x,-u)$ are all limited for each $x, u \in X$.

Now Question 2 presented in Section 1 is completely answered. Let us answer Question 3 by the following result, which shows us that the uniqueness of a minimal approximate Hessian for a continuously Gâteaux differentiable function is not true in general, say if the underlying space is not one-dimensional.

THEOREM 2.3. Let $f: X \rightarrow R$ be a continuously Gâteaux differentiable function, then the following statements are true.

(i) If $f$ admits an approximate generalised Hessian at $x$ for $u$, then the minimal approximate Hessian for $f$ at $x$ for $u$ is unique if and only if $u \neq 0$ and $X$ is one dimensional.

(ii) If $f$ admits a weak approximate generalised Hessian at $x$ for $u$, then the minimal weak approximate Hessian for $f$ at $x$ for $u$ is unique if and only if $u \neq 0, X$ is one dimensional, and there is at most one point in $A \cap B$.

Proof. (i) If $u=0$, Proposition 2.1 (iv) shows that the minimal approximate Hessians for $f$ at $x$ for $u$ are not unique. If $u \neq 0$ and $X$ is not one dimensional, both $\left\{x^{*} \in X^{*}:\left\langle x^{*}, u\right\rangle=f_{++}^{D D}(x, u)\right\}$ and $\left\{x^{*} \in X^{*}:\left\{x^{*}, u\right\rangle=f_{++}^{D D}(x,-u)\right\}$ are not single-point sets. So, by Theorems 2.1 and 2.2, the minimal approximate Hessians for $f$ at $x$ for $u$ are not unique.

On the other hand, if $u \neq 0$ and $X$ is one dimensional, $\left\{x^{*} \in X^{*}:\left\langle x^{*}, u\right\rangle=\right.$ $\left.f_{++}^{D D}(x, u)\right\},\left\{x^{*} \in X^{*}:\left\langle x^{*}, u\right\rangle=f_{++}^{D D}(x,-u)\right\},\left\{x^{*} \in X^{*}:\left\langle x^{*}, u\right\rangle=f_{-+}^{D D}(x, u)\right\}$ and $\left\{x^{*} \in X^{*}:\left\langle x^{*}, u\right\rangle=f_{-+}^{D D}(x,-u)\right\}$ are all single-point sets, which together with Theorems 2.1 and 2.2 imply that the minimal approximate Hessian for $f$ at $x$ for $u$ is unique.

(ii) This statement follows from Theorem 2.2 (i) and a slight modification of the proof of (i). The proof is complete.

\section{Minimal approximate Hessians for $C^{1,1}$ functions}

The generalised upper and lower second-order directional derivative and generalised Hessian for a $C^{1.1}$ function $f: X \rightarrow R$ at $x$ in the sense of Michel-Penot are 
given as follows:

$$
\begin{aligned}
& f_{+}^{\infty}(x ; u, v)=\sup _{z \in X} \limsup _{s \downarrow 0} \frac{\langle\nabla f(x+s z+s u), v\rangle-\langle\nabla f(x+s z), v\rangle}{s}, \\
& f_{-}^{\infty}(x ; u, v)=\inf _{z \in X} \liminf _{s \downarrow 0} \frac{\langle\nabla f(x+s z+s u), v\rangle-\langle\nabla f(x+s z), v\rangle}{s}, \\
& \partial^{\infty} f(x)(u)=\left\{x^{*} \in X^{*}: f_{-}^{\infty}(x ; u, v) \leq\left\langle x^{*}, v\right\rangle \leq f_{+}^{\infty}(x ; u, v) \text { for each } v \in X\right\} .
\end{aligned}
$$

Moreover, the following formulas hold for all $x, u \in X$.

$$
\begin{aligned}
f_{++}^{D D}(x, u), f_{-+}^{D D}(x, u) & \in\left\{\alpha: f_{-}^{\infty}(x ; u, u) \leq \alpha \leq f_{+}^{\infty}(x ; u, u)\right\} \\
f_{-}^{\infty}(x ; u, v) & =\min \left\{\left\langle x^{*}, v\right): x^{*} \in \partial^{\infty} f(x)(u)\right\} \text { for each } v \in X \\
f_{+}^{\infty}(x ; u, v) & =\max \left\{\left\langle x^{*}, v\right\rangle: x^{*} \in \partial^{\infty} f(x)(u)\right\} \text { for each } v \in X
\end{aligned}
$$

The definitions and formulas for the $C^{1,1}$ functions mentioned above can be found or derived from those in $[14,23]$.

Note that (3.1) implies that $\partial^{\circ \circ} f(x)(u)$ can be chosen as an approximate generalised Hessian for $f$ at $x$ for $u$, so there always exists a minimal approximate Hessian in $\partial^{\infty} f(x)(u)$ by Remark 2.1 (i). Then we may ask naturally: when can $\partial^{\infty} f(x)(u)$ itself be a minimal approximate Hessian? This is Question 1 from Section 1 and the following theorem gives the answer.

THEOREM 3.1. Let $f: X \rightarrow R$ be a $C^{1,1}$ function and $x, u \in X$. Then $\partial^{\circ \circ} f(x)(u)$ is a minimal approximate Hessian for $f$ at $x$ for $u$ if and only if $\partial^{\circ \circ} f(x)(u)$ is $a$ compact set of a one-dimensional subspace of $X^{*}$, and

$$
\begin{aligned}
& f_{+}^{\infty}(x ; u, u)=\max \left\{f_{++}^{D D}(x, u), f_{-+}^{D D}(x,-u)\right\} \\
& f_{-}^{\infty}(x ; u, u)=\min \left\{f_{++}^{D D}(x, u), f_{-+}^{D D}(x,-u)\right\}
\end{aligned}
$$

Proof. If $\partial^{\infty} f(x)(u)$ is a minimal approximate Hessian for $f$ at $x$ for $u$, we have two cases.

Case a. If $f_{++}^{D D}(x, u)=f_{-+}^{D D}(x,-u)$, Theorem 2.1 shows that a minimal approximate Hessian for $f$ at $x$ for $u$ is a single-point set $\left\{x^{*}\right\}$, where $x^{*} \in X^{*}$ s.t. $\left\langle x^{*}, u\right\rangle=f_{++}^{D D}(x, u)$. So $\partial^{\infty} f(x)(u)$ is a compact set of a one dimensional subspace of $X^{*}$. Moreover, by (3.1), we have $f_{-}^{\infty}(x ; u, u)=f_{++}^{D D}(x, u)=f_{-+}^{D D}(x,-u)=$ $f_{+}^{\infty}(x ; u, u)$. Thus (3.4) and (3.5) hold.

Case b. If $f_{++}^{D D}(x, u) \neq f_{-+}^{D D}(x,-u)$, Theorem 2.1 shows that a minimal approximate Hessian for $f$ at $x$ for $u$ is a convex hull $\operatorname{co}\left\{x_{1}^{*}, x_{2}^{*}\right\}$, where $x_{1}^{*}, x_{2}^{*} \in X^{*}$ s.t. $\left\langle x_{1}^{*}, u\right\rangle=f_{++}^{D D}(x, u)$ and $\left\langle x_{2}^{*}, u\right\rangle=f_{-+}^{D D}(x,-u)$. Thus $\partial^{\circ 0} f(x)(u)$ is a compact set of a one dimensional subspace of $X^{*}$. Furthermore, (3.1)-(3.3) implies (3.4) and (3.5). 
On the other hand, if $\partial^{\infty} f(x)(u)$ is a compact set of a one-dimensional subspace of $X^{*}$ and (3.4) and (3.5) hold, we also have two cases.

Case 1. If $f_{++}^{D D}(x, u)=f_{-+}^{D D}(x,-u), \partial^{\circ 0} f(x)(u)$ is single-point, and hence $\partial^{\circ \circ} f(x)(u)$ is a minimal approximate Hessian of $f$ at $x$ for $u$.

Case 2. If $f_{++}^{D D}(x, u) \neq f_{-+}^{D D}(x,-u)$, there exist a unique $x_{1}^{*} \in X^{*}$ and a unique $x_{2}^{*} \in X^{*}$ s.t. $\left\langle x_{1}^{*}, u\right\rangle=f_{+}^{\infty}(x ; u, u)$ and $\left\langle x_{2}^{*}, u\right\rangle=f_{-}^{\infty}(x ; u, u)$. Then it follows from (3.2)-(3.5) that $\partial^{\infty} f(x)(u)=c o\left\{x_{1}^{*}, x_{2}^{*}\right\}$, which implies that $\partial^{\infty} f(x)(u)$ is a minimal approximate Hessian for $f$ at $x$ for $u$ by Theorem 2.1. This completes the proof.

Since in a reflexive Banach space $X$, the mapping $x \rightarrow \partial^{\infty} f(x)(u)$ is singlevalued for each $u \in X$ if and only if $f$ is twice weakly Gâteaux differentiable at $x$ (see $[14,23]$ ), we have the following corollary by Theorem 3.1 immediately.

COROLlary 3.1. Let $f: X \rightarrow R$ be twice weakly Gâteaux differentiable at $x \in X$ , with Banach space $X$ reflexive, then $\partial^{\infty} f(x)(u)$ is a minimal approximate Hessian for $f$ at $x$ for $u$.

\section{Applications}

4.1. Generalised Taylor's expansions and convexity We now apply a minimal approximate Hessian to give the following modified version of the generalised Taylor's expansions given in [14] for continuously Gâteaux differentiable functions.

THEOREM 4.1. Suppose a continuously Gâteaux differentiable function $f: X \rightarrow R$ admits a weak approximate generalised Hessian at each $z \in X$. Then for each $x, y \in X$, there exists $\xi \in(x, y)$ (the open line segment from $x$ to $y$ ) s.t.

$$
f(y) \in f(x)+\langle\nabla f(x), y-x\rangle+\frac{1}{2}\left\langle\partial_{m}^{w w} f(\xi)(y-x), y-x\right\rangle .
$$

PROOF. The proof follows immediately from [14, Theorem 4.1] and Remark 2.1 (i).

By applying this generalised Taylor's expansion, we characterise the convexity of a continuously Gâteaux differentiable function in terms of a minimal approximate Hessian and generalised derivative.

THEOREM 4.2. Suppose a continuously Gâteaux differentiable function $f: X \rightarrow R$ admits a weak approximate generalised Hessian at each $x \in X$. Then the following statements are equivalent:

(i) $f$ is convex.

(ii) $f_{-}^{\infty}(x ; u, u) \geq 0$ for each $x, u \in X$. 
(iii) $\min \left\{\left\langle x^{*}, u\right\rangle: x^{*} \in \partial_{m}^{w w}(x)(u)\right\} \geq 0$ for each $x, u \in X$.

Proof. (i) $\Rightarrow$ (ii). Since $f$ is convex, $\langle\nabla f(y)-\nabla f(x), y-x\rangle \geq 0$ for each $x, y \in X$, and then

$$
f_{-}^{\infty}(x ; u, u)=\inf _{z \in X} \liminf _{s \downarrow 0} \frac{\langle\nabla f(x+s z+s u), u\rangle-\langle\nabla f(x+s z), u\rangle}{s} \geq 0,
$$

for each $x, u \in X$.

(ii) $\Rightarrow$ (iii). Note that (ii) and (3.1) imply $f_{-+}^{D D}(x, u) \geq 0$ for each $x, u \in X$. Then $f_{++}^{D D}(x, u) \geq f_{-+}^{D D}(x, u) \geq 0$ for each $x, u \in X$. Hence (iii) holds by Theorem 2.2 .

(iii) $\Rightarrow$ (i). By Theorem 4.1, for each $x, u \in X$, there exists $\xi \in(x, y)$ and $x^{*} \in \partial_{m}^{w w} f(\xi)(y-x)$ s.t.

$$
f(y)=f(x)+\langle\nabla f(x), y-x\rangle+\frac{1}{2}\left(x^{*}, y-x\right\rangle,
$$

which together with (iii) implies

$$
f(y) \geq f(x)+\langle\nabla f(x), y-x\rangle \text { for each } x, y \in X .
$$

Hence $f$ is convex.

REMARK 4.1. By [21, Theorem 3.1], we know that, although we have (3.1), the condition (ii) in Theorem 4.2 is equivalent to the condition below:

(iv) $f_{-+}^{D D}(x, u) \geq 0$ for each $x, u \in X$,

provided that the continuously Gâteaux differentiable function $f: X \rightarrow R$ admits a weak approximate generalised Hessian at each $x \in X$. In particular, for a $C^{1,1}$ function $f$, the condition (iii) in Theorem 4.2 is equivalent to

(v) $\min \left\{\left\langle x^{*}, u\right\rangle: x^{*} \in \partial^{\circ} f(x)(u)\right\} \geq 0$ for each $x, u \in X$.

This can also be derived from [14, Corollary 4.1$]$.

4.2. Second-order optimality conditions Here we present some second-order necessary and sufficient optimality conditions, by using minimal approximate Hessians, so that the range of the feasible points can be chosen as "small" as possible. This is important for optimisation problems. Consider the following optimisation problem:

(P) Minimise $f(x)$ subject to $x \in X$,

where $f: X \rightarrow R$ is a continuously Gâteaux differentiable function.

It follows from Remark 2.1 (i) that Theorems 5.1-5.3 in [14] can be modified immediately as the following Theorems 4.3 and 4.4, where we just need to consider the "smaller" feasible point set $\partial_{m}^{a a} f(x)(u)$ or $\partial_{m}^{w w} f(x)(u)$ rather than the whole $\partial^{a a} f(x)(u)$ or $\partial^{w w} f(x)(u)$. 
THEOREM 4.3. Suppose $f$ admits an approximate generalised Hessian at $x \in X$ for each $u \in X$.

(i) If $x$ is a local minimum of the problem (P), then $\nabla f(x)=0$ and for each $u \in X$ there exists $x^{*} \in \partial_{m}^{a a} f(x)(u)$ such that $\left\langle x^{*}, u\right\rangle \geq 0$.

(ii) If $x$ is a local maximum of the problem (P), then $\nabla f(x)=0$ and for each $u \in X$ there exists $x^{*} \in \partial_{m}^{a a} f(x)(u)$ such that $\left\langle x^{*}, u\right\rangle \leq 0$.

THEOREM 4.4. Let $\bar{x} \in X$. Assume that $f$ admits a weak approximate generalised Hessian at each $x$ near $\bar{x}$ for each $u \in X$. If $\nabla f(\bar{x})=0$ and for $0<\alpha<1$, each $u \in X$ with $u \neq 0$, the following holds:

$$
\left\langle x^{*}, u\right\rangle \geq 0 \text { for all } x^{*} \in \partial_{m}^{w w} f(\bar{x}+\alpha u)(u)
$$

and so $\bar{x}$ is a local minimum of the problem (P). Furthermore, if the inequality (4.1) is strict, $\bar{x}$ is a strict local minimum of the problem $(\mathrm{P})$.

\section{Acknowledgements}

The authors are grateful to the referees for their helpful comments. This paper is supported by the National Natural Science Foundation of China.

\section{References}

[1] A. Ben-Tal and J. Zowe, "A unified theory of first and second order conditions for extremum problems in topological vector spaces", Math. Programming Study 19 (1982) 39-76.

[2] J. V. Burke, "Second-order necessary and sufficient conditions for convex composite NDO", Math. Programming 38 (1987) 287-302.

[3] J. V. Burke and R. A. Poliquin, "Optimality conditions for nonfinite-valued convex composite functions", Math. Programming Ser. B 57 (1992) 103-120.

[4] W. L. Chan, L. R. Huang and K. F. Ng, "On generalized second-order derivatives and Taylor expansions in nonsmooth optimization", SIAM J. Control Optim. 32 (1994) 591-611.

[5] R. W. Chaney, "Second-order directional derivatives for nonsmooth functions", J. Math. Anal. Appl. 128 (1987) 495-511.

[6] F. H. Clarke, Optimization and nonsmooth analysis (Wiley-Interscience, New York, 1983).

[7] R. Cominetti and R. Correa, "A generalized second-order derivative in nonsmooth optimization", SIAM J. Control and Optim. 28 (1990) 789-809.

[8] R. Correa, A. Jofre and L. Thibault, "Characterization of lower semi-continuous convex functions", Proc. Amer. Math. Soc. 116 (1992) 67-72.

[9] A. V. Fiacco, "Second-order sufficient conditions for weak and strict constrained minima", SIAM J. Appl. Math. 16 (1968) 105-108.

[10] R. Fletcher, Practical methods of optimization (John Wiley, New York, 1987). 
[11] J. B. Hiriart-Umuty, J. J. Strodiot and V. Hein Nguyen, "Generalized Hessian matrix and secondorder optimality conditions for problems with $C^{1.1}$ data", Appl. Math. Optim. 11 (1984) 43-56.

[12] A. D. Ioffe, "Necessary and sufficient conditions for a local minimum. Part 3: Second-order conditions and augmented duality", SIAM J. Control Optim. 17 (1979) 266-288.

[13] V. Jeyakumar and X. Q. Yang, "Convex composite minimization with $C^{1,1}$ functions", J. Optim. Theory Appl. 86 (1995) 631-648.

[14] V. Jeyakumar and X. Q. Yang, "Approximate generalized Hessians and Taylor's expansions for continuously Gâteaux differentiable functions", Non. Anal. T. M. A. 36 (1999) 353-368.

[15] H. Kawasaki, "An envelope-like effect of infinitely many inequality constrains on second-order necessary conditions for minimization problems", Math. Programming 41 (1988) 73-96.

[16] P. Michel and J.-P. Penot, "A generalized derivative for calm and stable functions", Differential Integral Equations 5 (1992) 433-454.

[17] Z. Pales and V. Zeidan, "Generalized Hessian for $C^{1,1}$ functions in infinite dimensional normed spaces", Math. Programming 74 (1996) 59-78.

[18] L. Qi, "Superlinearly convergent approximate Newton methods for $L C^{1}$ optimization problems", Math. Programming 64 (1994) 277-294.

[19] R. T. Rockafellar, "First- and second-order epidifferentiability in nonlinear programming", Trans. Amer. Math. Soc. 307 (1988) 75-108.

[20] R. T. Rockafellar, "Second-order optimality conditions in nonlinear programming obtained by way of epi-derivatives", Math. Oper. Res. 14 (1989) 462-484.

[21] X. Q. Yang, "Generalized second-order characterizations of convex functions", J. Optim. Theory Appl. 82 (1994) 173-180.

[22] X. Q. Yang, "Second-order conditions in $C^{1,1}$ optimization with applications", Numer. Funct. Anal. Optim. 14 (1993) 621-632.

[23] X. Q. Yang and V. Jeyakumar, "Generalized second-order directional derivatives and optimization with $C^{1,1}$ functions", Optimization 26 (1992) 165-185. 